\title{
An Artificial Intelligence Based Glucometer for Diabetic Patients using Urinal Analysis
}

\author{
S. Geetha and V. Lakshminarayanan
}

\begin{abstract}
There are nearly about 50.8 million people in the world who have diabetes and maintaining the blood sugar level of diabetic patients is very important. The diabetic patient regularly monitor their glucose level by pricking the finger for taking blood samples 4-5 times a day and controls their sugar level by taking appropriate dosage of medicine. An artificial intelligence based image processing application has been developed, which non-invasively measures the glucose concentration present in the urine sample of a person and hence the equivalent blood glucose level of that person is inferred. Blood sugar level of a person from his/her urine sample has been monitored by noting the colour change of the test sample, when it is reacted with Benedict's reagent. The colour change of the sample is identified with the help of the camera and displays the result in the form of hue (predominant colour) value. This measurement has become possible by training the neural network using the hue value as the input vector and the glucose value as the test vector. A linear relationship has been obtained successfully with an accuracy of about $96.93 \%$.
\end{abstract}

Keywords--- Benedict's Reagent, Colour Sensor, Neural Network, HSI Colour Format, Diabetes Mellitus, Hue Value

\section{INTRODUCTION}

$\mathrm{I}^{2}$ is estimated that diabetics afflicts over 50.8 million people worldwide due to poor diet and obesity. It is not completely curable, but it can be controllable to the great extend. So it is the right time to develop a technology that helps to control diabetics among present society. Present day technologies limit such monitoring due to high cost of test strips and severe pain involved. A patient with hyperglycemia needs to prick their fingers many times per day to monitor their glucose concentration. So that patients affect from mental stress and it is of high cost. To overcome these problems, it is essential to go for non-invasive technique. In ancient days, people measure their glucose level by taking a urine sample and heat it with Benedict's reagent. The colour change of the sample gives the approximate sugar level. The urine sugar level is just a reflection of how high the blood glucose was, and how long it was above the renal threshold. With the help of the ancient system along with the advancement in

S. Geetha, PG Scholar, Dr.Mahalingam College of Engineering and Technology, Pollachi, India. E-mail: sgeethakec@gmail.com

V. Lakshminarayanan, Assistant Professor/EEE, Dr.Mahalingam College of Engineering and Technology, Pollachi, India. E-mail: vlnaarayan@gmail.com

DOI: $10.9756 /$ BIJPSIC.4278 technology, this non invasive blood sugar monitoring device is modeled.

Diabetes mellitus is a deficiency in which the body cannot regulate the amount of glucose (sugar) in the blood [1]. The glucose present in the body provides the energy to perform person's daily activities. The liver performs the conversion of food into glucose and the glucose is then released into the bloodstream. The blood glucose level of a healthy person is regulated by hormones like insulin. Insulin is produced by the pancreas, a small organ that lies between the stomach and liver. The pancreas also releases some other enzymes which help to digest the food. Insulin is very helpful in moving the glucose out of the blood into the cell throughout the body, where it is used as a fuel [2].

People with diabetes either do not produce enough insulin or cannot use insulin properly in day-to-day life or both. In diabetes, glucose cannot move efficiently into the cells, so glucose level in the blood remains high. This starves the cells which needs the fuel (i.e) glucose. It also harms the organs and tissues exposed to high glucose levels.

There are different forms of diabetes. Type 1 diabetes is a type of diabetes in which the body cells stop producing insulin or it produces too little insulin to regulate the blood sugar level [3]. Type 1 diabetes is usually referred to as insulin-dependent diabetes. It usually occurs at the older age due to damage in the pancreas by alcohol or diseases. Type 2 diabetes is a type of diabetes in which the insulin produced by the pancreas is partially or completely not used by the blood cells [4]. It is usually referred to as insulin resistance or insulin-independent diabetes. This type of diabetes does occur at the younger age and so some people require insulin therapy. It is usually controlled with diet, weight loss, exercise and oral medicines.

The main symptoms of the diabetes are loss of weight, frequent urination, increased thirst and increased hunger [5]. The symptoms may develop rapidly in type 1 diabetes; while they usually slower or it may be absent in type 2 diabetes [6]. Prolonged hyper glycemia leads to changes in the shape of a lens present in eye, resulting in blurred vision. Type 1 diabetes can easily be identified by rapid vision change whereas type 2 diabetes is generally gradual. The skin rashes also occurs in diabetes, which is commonly known as diabetic dermadromes[7][8].

Conventionally diabetes can be diagnosed by blood or urine of a person. There are two main categories for the measurement of glucose level. Invasive technique, which monitors blood glucose level by pricking the hand to take blood samples or implanting sensors into human body for continuous measurement [9]. This technique is used in present 
laboratories. The main disadvantage of this technique is test strips are much costlier and patient needs to afford a syringe of blood. Non invasive technique, it's like a hell to heaven for diabetic patients. Non-invasive technique, which measures the glucose concentration by taking urine sample instead of pricking the finger for taking the blood samples [10]. This paper mainly deals with the non-invasive technique which continuously monitors the blood glucose level by taking the urine sample of a person. The main advantage of this technique is pain free and it is of much affordable cost. In this proposal the design and experimental set up for blood sugar measuring device is discussed in detail in section II and also the image processing and neural network processing is used and the test results obtained are reported in following sections III and IV in detail.

\section{EXPERIMENTAL SETUP}

The experimental set up of the system is shown in the figure 1. In figure 1, there are three ports namely inlet port, reagent port and outlet port. The inlet port opens to collect the urine sample. The reagent port collects the Benedict's reagent. The heater is used to boil the mixer up to the boiling point automatically, which is controlled by computer. The colour change of the mixer is sensed by Camera and after sensing the colour, the waste is removed through the outlet port. The colour sensed by the camera is then processed in personnel computer by an artificial intelligence based system. The entire process is explained with the help of a flow diagram which is shown in figure 2 .

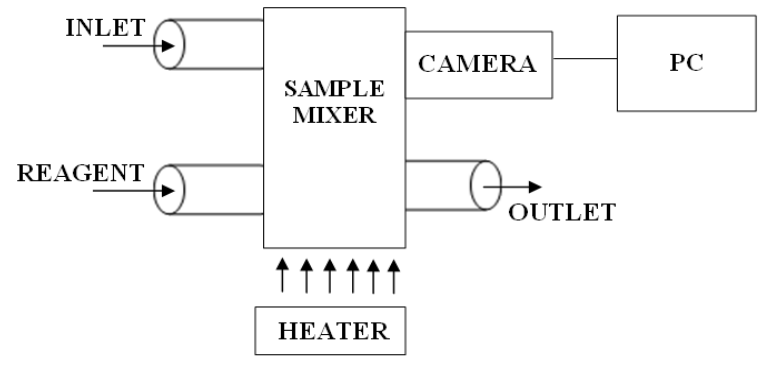

Figure 1: Experimental Setup

\section{MATERIALS AND METHODS}

The entire work is an amalgamation of mechanical and control engineering, chemistry, image processing and neural network. The next section will explain the system details.

\subsection{Benedict's Reagent}

Benedict's solution acts as a reagent to find the blood sugar level. Benedict's solution is used to find the sugar level present in the urine which gives the equivalent blood sugar value [11]. Benedict's reagent is composed of copper sulphate, sodium carbonate and sodium citrate. When Benedict's reagent is reacted with urine sample, its colour changes based on the amount of sugar level. The healthy persons normally have the blood glucose concentration in the range of 70$120 \mathrm{mg} / \mathrm{dl}$. Formation of red, green and yellow precipitate denotes the positive result for glucose test. Blue colour denotes the NIL sugar that means blood sugar level is less than
$180 \mathrm{mg} / \mathrm{dl}$. Green precipitate indicates the blood glucose level is appropriate to $180-220 \mathrm{mg} / \mathrm{dl}$.

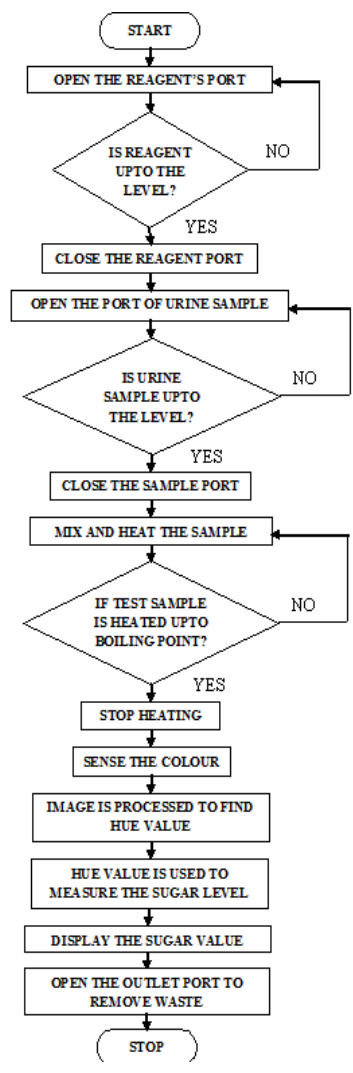

Figure 2: Flow Diagram

Yellow precipitate denotes the glucose concentration is appropriate to $221-280 \mathrm{~g} / \mathrm{dl}$. Red precipitate represents the blood sugar level in the range of $281-350 \mathrm{mg} / \mathrm{dl}$. Brick red colour denotes the sugar level is very high above $350 \mathrm{mg} / \mathrm{dl}$.

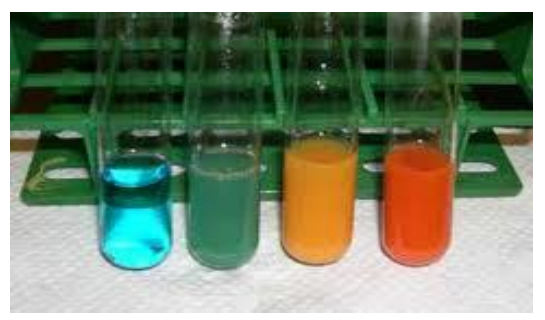

Figure 3: Different Colours of Urine Sample Reacted with the Benedict's Reagent

Figure 3 shows the different colours of the urine sample reacted with the Benedict's reagent[12]. In olden days the blood sugar value is predicted with the help of these colour changes. In this paper with the help of this reference value, an accurate blood sugar value is easily identified by capturing and processing the image obtained.

\subsection{System Description}

Figure 4 shows the block diagram of the system. The urine is collected in a test tube. Benedict's reagent $(5 \mathrm{ml})$ is added to the collected sample and it is mixed thoroughly to get a test sample. Holding the test tube with holder and heat it up to the boiling point with the help of a heater. Then allow the solution 
to cool down for few minutes, while cooling the mixture changes its colour. The colour of the mixture which serves as a guide to the amount of sugar in the urine which infer the equivalent blood sugar level [13]. The colour change is then captured by colour sensor and the image is loaded into the PC for further calculation using artificial intelligence technique. The solution is then drained out as a waste.

\subsection{Image Processing}

The colour change obtained after heating the mixer is captured by camera. The colour change obtained is uniform but the image captured by the colour sensor is generally noise. In order to obtain the original image, convolution based filtering is used. This type of filtering removes the noise from the image and gives the original image.

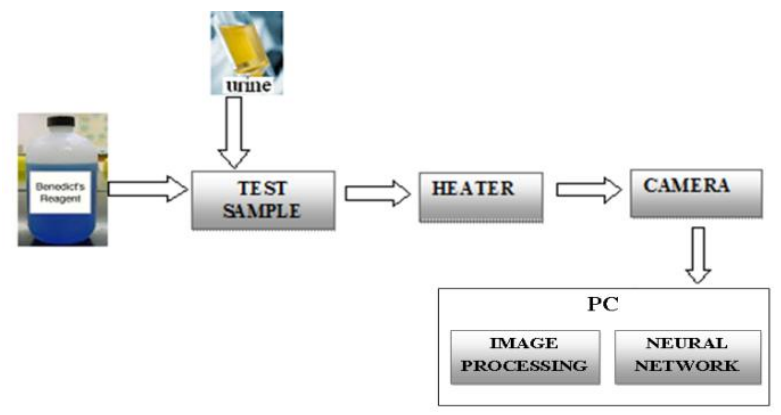

Figure 4: Block Diagram of the System

Convolution based filtering is used separately on the Red, Green and Blue components of the sensed image by equation 1

$$
g(x, y)=\sum_{u=-a v=-b}^{a} \sum^{b} w(u, v) f(x+u, y+v)
$$

where, $\mathrm{w}$ is the convolution mask of size ( $\mathrm{mxn})$,

$\mathrm{f}$ is the original image captured with noise

$\mathrm{x}$ and $\mathrm{y}$ is the row and column indexes of the image.

The convolution mask of size (mxn) have the coefficient values equal to $1 /(\mathrm{mxn})$. The $\mathrm{m}$ values ranges from $-\mathrm{a}$ to $+\mathrm{a}$ and the $n$ values ranges from $-b$ to $+b$, where $a$ and $b$ are nonnegative integers. The coefficient value $\mathrm{w}(0,0)$ coincides with the image value $f(x, y)$, such that the convolution mask is centered at $(\mathrm{x}, \mathrm{y})$ position of the sensed, where the computation takes place. $\mathrm{g}(\mathrm{x}, \mathrm{y})$ finally gets the response of the filter. After applying the filter to the noisy image the red, blue and green components are assembled again to build the original image. The sensed image is shown in the figure 5.
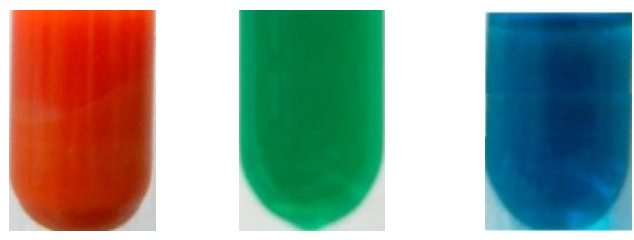

Figure 5: The Sensed Original Image

The next step is to extract the colour information from the sensed image. The original image contains only the information about the primary colours that is RGB components. The main drawback of using RGB components is that it not only contains the colour information but also the colour information but also contains the colour intensity.

The RGB values are different for different intensities i.e it is different for light blue, dark blue and navy blue. With the RGB values the original colour of the image is not easily identified. To overcome this problem different colour format has been used. The HSI colour format is suggested to find the predominant colour of the original image, where $\mathrm{H}$ stands for Hue i.e pure colour, S stands for Saturation i.e the degree by which the pure colour is diluted by the white colour and I stands for the Intensity i.e grey level value.

The RGB to HSI colour format conversion is required to find the pure colour of an image. The HSI colour format conversion is performed using following equations.

$$
\begin{gathered}
H=\left\{\begin{array}{l}
\theta \ldots \ldots . ., \text { if } . . B \leq G \\
360-\theta, \text { otherwise }
\end{array}\right\} \\
\theta=\cos ^{-1}\left\{\frac{\frac{1}{2}[(R-G)+(R-B)]}{\sqrt{[(R-G)(R-G)+(R-B)(G-B)}}\right\} \\
S=1-\frac{3}{(R+G+B)}[\min (R, G, B)] \\
I=\frac{1}{3}(R+B+G)
\end{gathered}
$$

In the HSI components, Saturation and Intensity should have values in the range $[0,1]$ and Hue should have values in the range $[0,360]$

Algorithm to find the Hue value matrix from the image.

RGB stands for Red, Green and Blue components of the image. " $\mathrm{H}$ " is a matrix which contains the Hue from an image. "Row" is a vector from a hue matrix. "minimum()" is a function which returns the minimum hue value.

Step 1: Equation 3 is applied to each pixel of the RGB components of an image and the Hue values that are obtained are stored in the $\mathrm{H}$ matrix.

Step 2: The middle element is found from the $\mathrm{H}$ matrix by sorting entire row's element and store it in a vector named "Row".

Step 3: Sort the "Row" vector and the middle element is taken as a Hue value.

Step 4: As already seen the Hue value varies from 0 to 360, hence

Actual Hue = minimum $($ Hue, 1-Hue $)$.

Step 5: Return the Actual Hue value.

Step 6: Stop.

\subsection{Deciding the Sugar Level}

It is very difficult to identify the pure colour from the hue value of an image. So some other techniques should be followed to overcome this problem. Neural network training pattern is used to solve this problem. Neural network is an artificial intelligence based method, which can be viewed as a neurons processing in a human brain[14]. Neural networks are a form of multiprocessor computer systems with simple 
processing elements and high degree of interconnections. There are many types of neural network models available. In this paper, back propagation model is adapted to train the network pattern.

Back propagation neural network is derived from the delta rule, in which extra hidden layers can be added to show the percentage of sugar more precisely. The input and the target vectors should be fed initially for training the network pattern. Training these networks is done by changing the weights of the unit depends on the error occurring at that unit. The weight change rule is developed from the perceptron learning rule. The output unit error is used to alter the weights on the output unit. The hidden layer errors can be calculated by back propagating the errors at the output unit and the hidden layer weights are altered with those errors. For each data set, the forward pass and backward pass is continued until the error become too low. Unlike other networks, in back propagation network the errors can be back propagated to the hidden layers, so that more accurate results can be obtained. The structure of the back propagation neural network was shown in figure 6.

In this project single layer net is used which means one input unit, one hidden layer and one output unit. To get the more accurate results hundreds of iterations should be done to achieve the target output. More number of iterations is to be made to correct the errors by forward pass and backward pass. The forward pass and the backward pass can be done using the following equations.

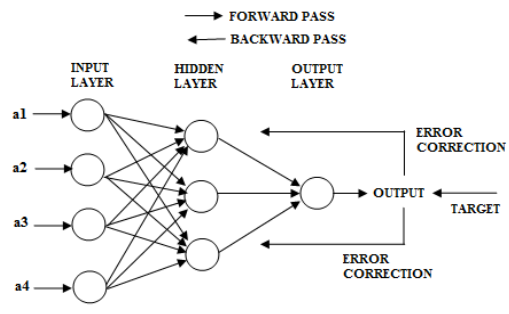

Figure 6: Back Propagation Neural Network

The forward pass:

Input to the hidden unit,

$$
\mathrm{Z}_{\mathrm{inj}}=\mathrm{V}_{0 \mathrm{j}}+\sum_{i} x_{i} v_{i j}
$$

Output of the hidden layer,

$$
\mathrm{Z}_{\mathrm{j}}=f\left(\mathrm{Z}_{\mathrm{inj}}\right)
$$

Table 1: Training Test Vector Sugar Measurement

Input to the output unit,

$$
\mathrm{Y}_{\text {ink }}=\mathrm{W}_{0 \mathrm{k}}+\sum_{j=1}^{p} Z_{j} W_{j k}
$$

To compute the output signal,

\begin{tabular}{|c|c|c|c|c|c|c|c|}
\hline \multicolumn{2}{|c|}{$\begin{array}{c}\text { RED } \\
\text { COLOUR }\end{array}$} & \multicolumn{2}{|c|}{$\begin{array}{l}\text { YELLOW } \\
\text { COLOUR }\end{array}$} & \multicolumn{2}{|c|}{$\begin{array}{l}\text { GREEN } \\
\text { COLOUR }\end{array}$} & \multicolumn{2}{|c|}{$\begin{array}{c}\text { BLUE } \\
\text { COLOUR }\end{array}$} \\
\hline Hue & $\%$ & Hue & $\%$ & Hue & $\%$ & Hue & $\%$ \\
\hline 0.007 & 100 & 0.039 & 20 & 0.184 & 10 & 0.417 & 20 \\
\hline 0.039 & 80 & 0.060 & 30 & 0.192 & 20 & 0.452 & 30 \\
\hline 0.060 & 70 & 0.082 & 40 & 0.214 & 70 & 0.481 & 60 \\
\hline 0.082 & 60 & 0.106 & 50 & 0.245 & 80 & 0.5 & 80 \\
\hline 0.106 & 50 & 0.127 & 60 & 0.272 & 100 & 0.510 & 100 \\
\hline 0.127 & 40 & 0.148 & 70 & 0.314 & 100 & 0.534 & 100 \\
\hline 0.148 & 30 & 0.166 & 90 & 0.333 & 100 & 0 & 0 \\
\hline 0.166 & 10 & 0.184 & 90 & 0.394 & 90 & 0 & 0 \\
\hline 0 & 0 & 0.192 & 80 & 0.417 & 80 & 0 & 0 \\
\hline 0 & 0 & 0.214 & 30 & 0.452 & 70 & 0 & 0 \\
\hline 0 & 0 & 0.245 & 20 & 0.481 & 40 & 0 & 0 \\
\hline 0 & 0 & 0 & 0 & 0.5 & 20 & 0 & 0 \\
\hline
\end{tabular}

$$
\mathrm{Y}_{\mathrm{k}}=f\left(\mathrm{Y}_{\mathrm{ink}}\right)
$$

The backward pass:

The error correction term of the output unit,

$$
\delta k=\left(T_{k}-Y_{k}\right) f^{\prime}\left(Y_{i n k}\right)
$$

Updating the weights and bias of the output unit,

$$
\begin{aligned}
& \Delta W_{j k}=\alpha \delta_{k} Z_{j} \\
& \Delta W_{0 k}=\alpha \delta_{k}
\end{aligned}
$$

Error correction term of the hidden unit,

$$
\begin{aligned}
& \delta_{i n j}=\sum_{k=1}^{m} \delta_{k} W_{j k} \\
& \delta_{j}=\delta_{i n j} f^{\prime}\left(Z_{\text {inj }}\right)
\end{aligned}
$$

Update the weight and bias of the hidden unit,

$$
\begin{aligned}
& \Delta V_{i j}=\alpha \delta_{j} x_{i} \\
& \Delta V_{0 j}=\alpha \delta_{j}
\end{aligned}
$$

Where $\mathrm{i}$ is the number of input vectors

$\mathrm{j}$ is the number of hidden vectors

$\mathrm{k}$ is the number of output vectors

$\mathrm{T}$ is the target unit

The equation (11) and (15) update the weights to reduce the errors. In blood glucose measuring technique by urine and Benedict's reagent, four colours test set is required. The sugar 
level is identified with the help of the four colours like blue, green, yellow and red. By majority voting technique, the training test set is formed and the final decision is to be made by this test vector.

The next session describes the algorithm to find the percentage of sugar present in the urine with the help of Hue value obtained. The input and the target vector are initially assigned to the neural network. The weights and bias is also randomly assigned. After training the network pattern to the minimum error value, the calculation for finding the sugar value has to be done.

Step 1: Assign the minimum and the maximum value of the input test vector.

Step 2: Set product value $=1$.

Step 3: Output $=$ bias + (activation function $*$ weights $)$.

Step 4: Find the ratio for the de-normalization.

ratio $=[\max -\min$ of the input vector $] /[\max -\min$ of the reference values taken]

Step 5: De-normalization process is done.

$\mathrm{Nt}=($ output + product value $) /$ ratio
Step 6: Final output $=\mathrm{Nt}+$ minimum reference value.

The trained test vector by majority voting technique for measuring the sugar value is shown in table 1 . The training data set is formed by taking the urine samples and blood samples from 1000 patients and the test vector is formed manually.

\section{CIRCUIT DESCRIPTION}

The circuit diagram of the proposed system is shown in figure 7. To automate the process, micro-controller is used to control the heater, timers and image capturing process. Figure 8 shows the image of the circuitry. The captured image is loaded into the $\mathrm{PC}$, the image is processed through image processing technique and the pure colour of the image is identified.

Then feed the colour of the image for processing the neural network. By using training test vectors, hue value obtained is converted into percentage of sugar value in that particular colour. The conversion process is done to convert the percentage of sugar into real sugar value in $\mathrm{mg} / \mathrm{dl}$. The conversion is done using neural network tool box in MATLAB.

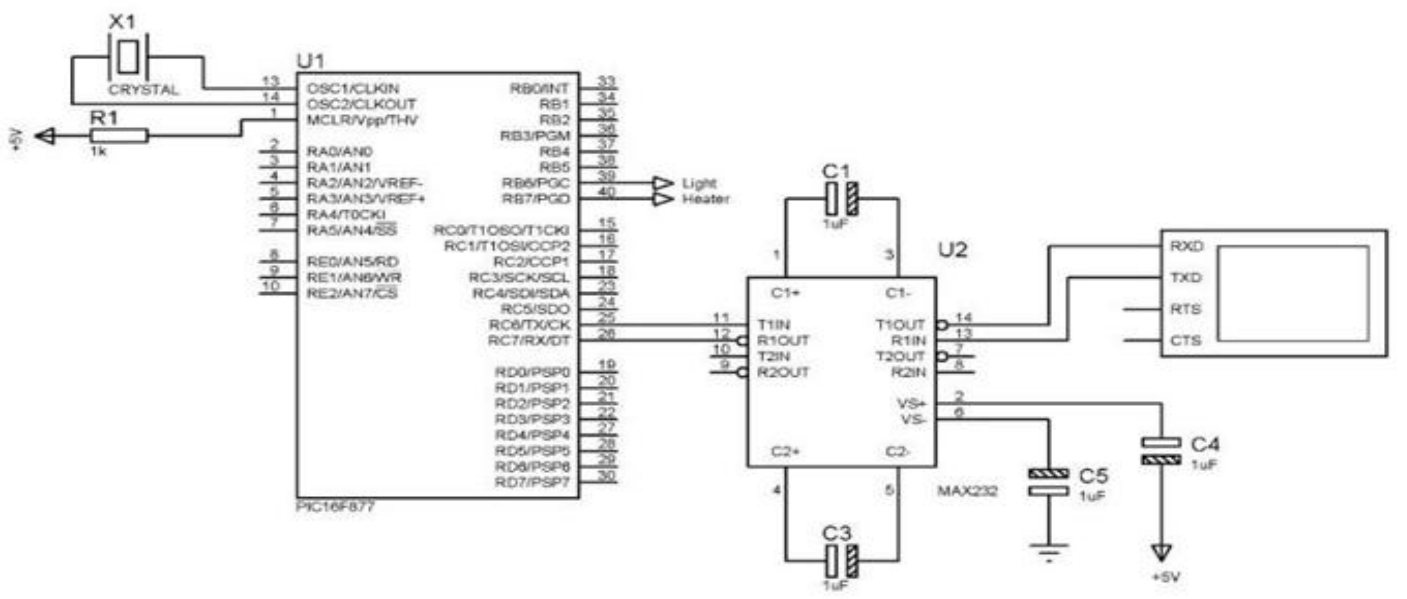

Figure 7: Circuit Diagram of the Proposed System

\section{SimUlation AND RESUlt}

The simulation for the artificial intelligence based image processing is done by MATLAB tool box. The simulation is used to extract the final hue value from the sensed image by image processing tool box and the extracted hue value is trained by the neural network toolbox to get the blood sugar value.

Figure 8 shows the graph of hue value of the test sample Vs percentage of sugar level of each colour image. The red vertical line shows the sugar level of red colour image which indicates the maximum glucose content. Yellow line shows the response of the yellow colour image which denotes the sugar value in the range of 221-280 mg/dl. Similarly Green and the Red line show the response of the corresponding image.

The algorithm used in this paper gives the more precise hue value. The hue value obtained for red colour image in figure 5 is 0.0277 . This pure value can be used to find the percentage of sugar value that is being present in the red image. The final output value for red colour image is 88.1713 which indicate the maximum amount of glucose in the blood. So, the final decision is that the blood sugar value is much higher for that particular patient and the appropriate dosage of medicine should be given.

For testing purpose nearly 200 samples are used in which 194 samples gives the satisfactory results. Thus the error rate is up to $4 \%$. In diagnostic centre, the accuracy is tested by taking both blood and urine samples from the diabetic patients. 


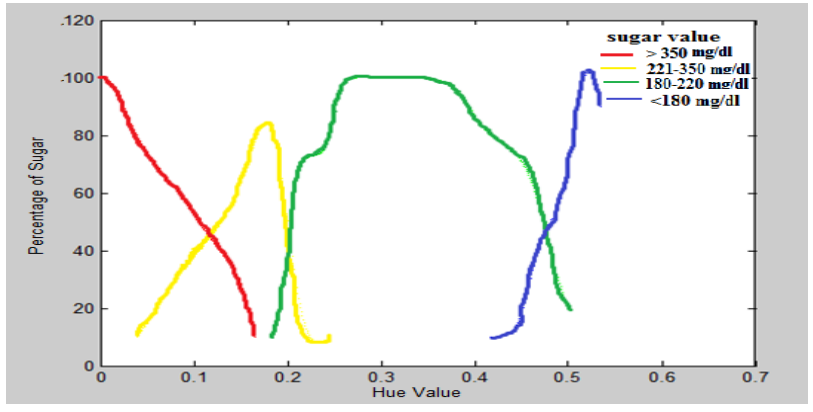

Figure 8: Hue value Vs Percentage of Sugar

\section{CONCLUSION AND DISCUSSION}

An artificial intelligence based image processing technique for glucose measurement is designed based on photometric principle. The major problem in medical field is to measuring the blood glucose level invasively. Everyone is looking for non-invasive devices to overcome that problem. This project aimed to use the non-invasive non-optical technique, which means taking the urine samples of the patient instead of taking the blood samples. An artificial intelligence based technique is used to find the most precise sugar value.

This system is very simple and efficient to detect the blood sugar level and also cost effective with no pain. It can reduce the probability of glaucoma, kidney problem, blurred vision, etc by early detection of high blood glucose level. This device can be improve and upgrade to produce a marketable product and usage for the user. Although this project is successfully developed and met the objective, however it is found that this system can be further improved by integrating it using a PIC microcontroller. The algorithm used for this approach is very simpler and provides the accuracy of about $96 \%$.

In future, the experimental setup is installed in real time and more accurate values can be obtained by training the neural network for each colour which indicates the different level of sugar in the blood. The training test vector for sugar measurement indicates the different percentage value of sugar level for each colour obtained. The module will be developed to find the most accurate glucose values from $30 \mathrm{mg} / \mathrm{dl}$ to $400 \mathrm{mg} / \mathrm{dl}$. The system developed is interfaced with the Computer to display the result in $\mathrm{mg} / \mathrm{dl}$. Future work of this system is to enhance the neural network based decision algorithms containing large training sets, so that the result will be more precise.

\section{REFERENCES}

[1] S. Amir, C. Rabin and A. Galatzer, 'Cognitive and behavioral determinants of compliance in diabetics', Health Soc. Work, Pp: 144$151,1990$.

[2] R.C. Bateman, J.A. Evans, "Using the glucose oxidase/peroxidase system in enzyme kinetics", J. Chem. Educ, Vol. 72, Pp: A240-A241, 1995.

[3] Ragnar Hanas, "Type 1 diabetes in children, adolescents and young adults", 3th Ed, Uddevalla, Sweden, 2007.

[4] Patrick English, Gareth Williams, "Type 2 Diabetes" London, UK, Martin Dunitz Ltd, 2001.

[5] Christine Brooker, "Human structure and function: nursing applications in clinical practice", 2nd Ed, 1997.
[6] Patrick English and Gareth Williams, 'Type 2 Diabetes' London, UK, Martin Dunitz Ltd, 2001.

[7] S. Amir, C. Rabin, A. Galatzer, "Cognitive and behavioral determinants of compliance in diabetics", Health Soc. Work, Pp. 144$151,1990$.

[8] N. Engl. J. Med., "The effect of intensive treatment of diabetes on the development and progression of long-term complications in insulindependent diabetes mellitus", The Diabetes Control and Complications Trial Research Group, Vol. 329, Pp. 977-986, 1993.

[9] Carlos F. Amaral, "Multiparameter techniques for non-invasive measurement of blood glucose", Sensors and actuators B, vol. 140, Pp.12-16, 2009.

[10] Ho Dong Parka, Kyoung Joung Leea, Hyung Ro Yoona, Hak Hyun Namb, "Design of a portable urine glucose monitoring system for health care", Computers in Biology and Medicine, Vol. 35, Pp. 275286, 2005.

[11] B.D. Lewis, 'Laboratory evaluation of the glucocard blood glucose test meter', Clin. Chem., Vol. 38, Pp. 2093-2095, 1992.

[12] Ahmad nadhil bin ngah, "Digital Urine Tester (Diabetes)".

[13] B.D. Lewis, "Laboratory evaluation of the glucocard blood glucose test meter", Clin. Chem. Vol. 38, Pp. 2093-2095, 1992.

[14] Ben Krose and Patrick van der Smagt, 'An Introduction to Neural Networks', eighth edition, 1996. 